ANOVA/Kruskal-Wallis, chi-square test and Pearson coefficient were used as appropriate. Logistic regression was used to identify independent associations with depression. Principal component analysis (PCA) with biplot and hierarchical cluster analysis (Euclidean distance) were done to evaluate the relationship of depression categories with QOL.

A Bonferroni corrected two-tailed 'p' value of $<0.025$ was considered statistically significant.

Results 224 patients were screened of which 208 were included. $151(72.6 \%)$ were males, and the most common aetiology was -idiopathic (150 [72.1\%]). Depression was seen in $125(60.1 \%)$ patients (figure 1a). Frequencies of depression categories are depicted in IDDF2018-ABS-0250 figure $1 \mathrm{~b}$. There was a significant positive correlation between depression score with a number of pain episodes $(\mathrm{r}=0.464 ; \mathrm{p}=0.02)$. Socio-economic status and continuous pain were independently associated with depression $(\mathrm{p}<0.0001$ and 0.02 respectively).

PCA demonstrated clustering of patients with and without depression. Biplot vectors showed the strongest negative correlation of the depression grades with global health $(p<0.0001)$, role function $(p=0.013)$, physical function $(p=0.009)$, emotional function $(p=0.004)$ and cognitive function $(p=0.017)$, while strongest positive correlation with fatigue $(p=0.001)$ and insomnia $(p=0.004)$ in the QORTC QLQ-30 (figure 2a). Clustering of the different categories of depression in the context of QOL domains was confirmed by the hierarchical clustering (Cophen. coeff. 0.719) (figure 2b).

Conclusions Continuous pain and socioeconomic status independently determine the development of depression in CP. Depression adversely impacts the global, functional, emotional and cognitive components of the QOL.
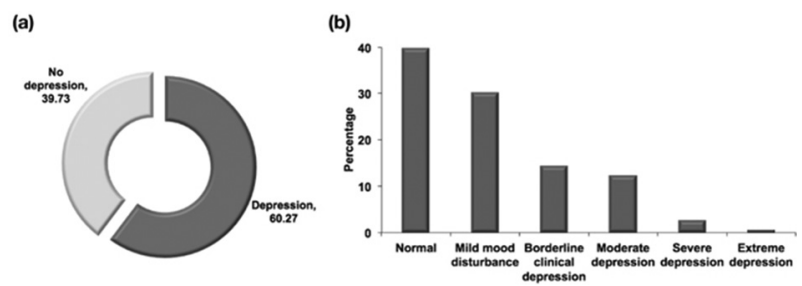

Abstract IDDF2018-ABS-0250 Figure 1 a, b (a)

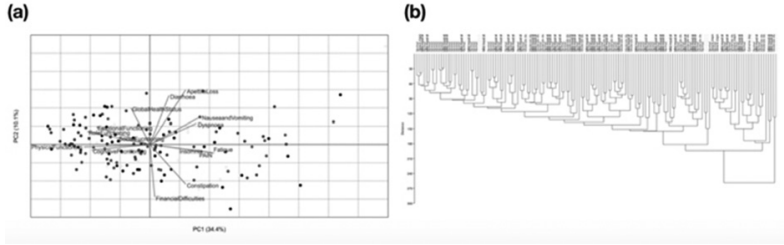

Abstract IDDF2018-ABS-0250 Figure 2 a, b

\section{IDDF2018-ABS-0251 DUCTAL CLEARANCE OF CALCULI DELAYS THE DEVELOPMENT OF DIABETES IN PATIENTS WITH IDIOPATHIC CHRONIC PANCREATITIS}

Rupjyoti Talukdar*, Manu Tandan, Sundeep Lakhtakia, Mohan Ramchandani, Rajesh Gupta, Rebala Pradeep. Asian Institute of Gastroenterology, India

\subsection{6/gutjnl-2018-IDDFabstracts. 174}

Background In this study, we evaluate the effect of pancreatic ductal clearance on its development in chronic pancreatitis (CP).

Methods Consecutive patients visiting the Pancreas Clinic between 1 st Aug, 2011-31stJul, 2012 were enrolled. Data on demography/disease progression/morphology (imaging)/treatment were recorded after verifying available documents. Thereafter patients were prospectively followed 6-mthly till June 2017. Patients who couldn't return were interviewed telephonically. Patients without complete data and those who lost to follow-up were excluded. Prospective data till June 2017 is presented.

Chi-square test was performed for categorical variables; Student's ' $t$ ' or the Mann Whitney U test was conducted for continuous variables. Binary logistic regression was performed to identify disease-related factors that imparted independent risk of development of diabetes. Kaplan-Meier survival analysis was used to compare the diabetes-free interval since onset of CP symptoms, and Cox-proportional hazards model to calculate the hazard ratio (HR) (95\% confidence interval[CI]). Twotailed 'p' value of $<0.05$ was considered statistically significant.

Results 644 patients were enrolled, of which 137 were excluded. Of the 507 analysed patients, $312(61.5 \%)$ had (a)

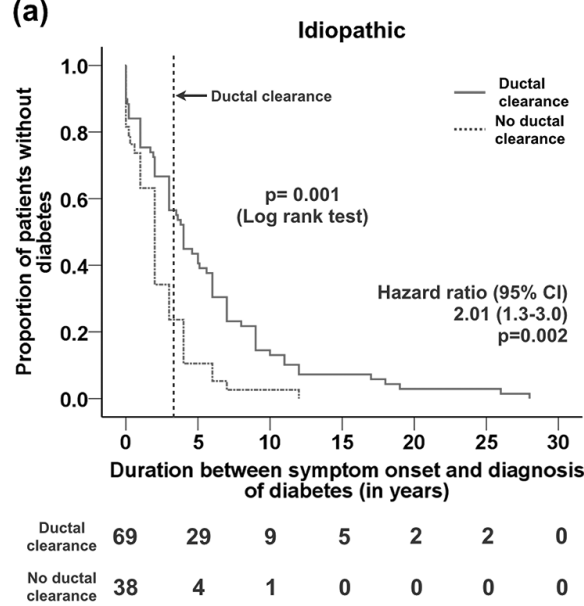

(b)

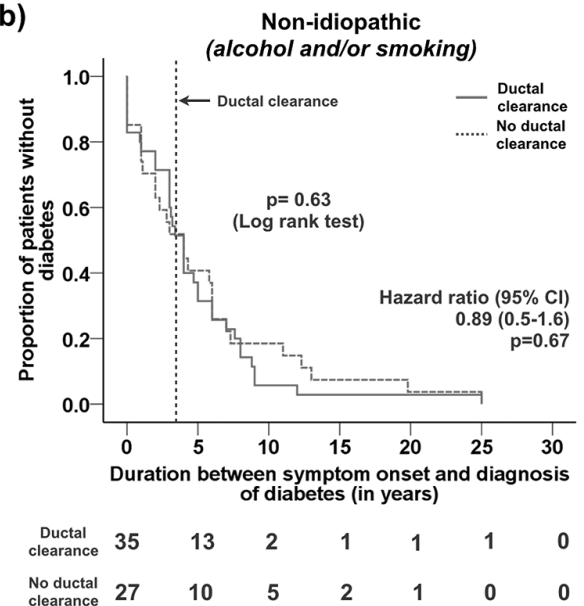

Abstract IDDF2018-ABS-0251 Figure 1 Kaplan Meier curve showing the development of diabetes 
idiopathic CP. 283 (55.8\%) patients underwent ductal clearance (ESWL/ERCP 232, drainage surgery 27, surgery following ESWL 24). 190 (37.5\%) patients developed diabetes. Mean(SD) duration between onset of CP symptoms and ductal clearance was 3.4 (3.2) yrs.

Alcohol intake and pancreatic ductal calculi were independently associated with the development of diabetes (OR [95\% CI] of 2.01 [1.16-3.47], p=0.03 and 2.04 [1.38-3.01], $\mathrm{p}<0.0001$ respectively). Kaplan-Meier estimate for diabetes free patients was significantly higher in the patients who underwent ductal clearance in patients with idiopathic CP ( $\mathrm{p}=0.001$ by Log-rank test), with a Hazard ratio (HR [95\% CI]) of $2.01(1.3-3.0), p=0.002$ (figure 1). This was not significantly different for patients with non-idiopathic (alcohol \pm smoking) CP.

Conclusions Pancreatic ductal calculi increase the risk of diabetes in CP. Early ductal clearance of stones could prolong the diabetes-free interval in patients with idiopathic CP.

\section{IDDF2018-ABS-0252 BENEFICIAL AND ANTI-INFLAMMATORY EFFECTS OF CINNAMALDEHYDE ON PATIENTS WITH FUNCTIONAL DYSPEPSIA}

Sumit Rajput*, Sukrat Sinha. School of Biotechnology, Guru Gobind Singh Indraprastha University, Dwarka, New Delhi, India

\subsection{6/gutjnl-2018-IDDFabstracts. 175}

Background Functional dyspepsia (FD) is a common functional gastrointestinal disorder with pain or discomfort in the upper abdomen as the main characteristic. The prevalence of FD worldwide varies between $5 \%$ and $11 \%$. This condition adversely affects attendance and productivity in the workplace. Cinnamaldehyde (CA) showed to have many pharmacological activities indicating anti-diabetic, cognitive enhancer, antiinflammatory etc. This study aimed at investigating the beneficial and anti-inflammatory effect of CA on the severity and frequency of symptoms in patients with FD.

Methods In total, 250 FD patients were included in this randomised double-blind trial, based on the ROME III diagnostic criteria, and they were divided into three intervention groups namely, CA, placebo and pantoprazole. Then, severity and frequency of symptoms during this twelve-week trial were measured. Obtained information was analysed using Chi-square test and repeated measures test.

Results In general, the severity and frequency of symptoms after the 12th week significantly decreased in the CA group as compared to the pantoprazole and placebo groups and continued to reduce by the end of the twelve weeks. General reduction of symptom severity and frequency in the pantoprazole group was significantly different from the placebo group by the end of the 4th and 12th weeks. With respect to each individual symptom, CA markedly improved symptoms, such as burning, pain, early satiation, fullness, bloating, belching and nausea, as compared to the placebo-treated group. Moreover, CA significantly improved symptoms, like vomiting, and nausea, except for pain, as compared to pantoprazole treated subjects.
Conclusions According to the results, CA, as traditional Indian remedy, was more effective than pantoprazole and placebo in reducing the symptoms in FD patients.

\section{IDDF2018-ABS-0253 A DEEP LEARNING METHOD FOR INTESTINAL POSITION LOCATING IN WCE}

'Liansheng Wang*, ${ }^{2}$ Qi Qiu. ${ }^{1} X i a m e n$ University, China; ${ }^{2}$ Xiamen Innovision Medical Technology Company, China

\subsection{6/gutjnl-2018-IDDFabstracts. 176}

Background In a wireless capsule endoscopy (WCE) abnormal automatic detection system, the same image content may have different meanings in different locations. For example, visible blood vessels are usual in ileum but abnormal in the duodenum and upper jejunum, and bile is normal in the duodenum but indicating bile reflux in the stomach, as shown in figure 1. Therefore, the essential first step of building this system is to locate the intestinal position of the image.

Methods To tackle this problem, a dense, connected convolution neural network (CNN) was adopted. The data were collected from several hospitals, consist of complete WCE videos of 16 patients, which has an average of 60000 images per video. Those images were classified into four classes: oesophagus, stomach, small intestine and others by a gastroenterologist. The images before oesophagus part and after small intestine part were excluded because the large intestine disease is not the goal of the WCE.

Results We used images of 10 videos (60\%) for training and the rest 6 videos $(40 \%)$ for testing. The performances are shown in table 1 .

\begin{tabular}{lccc}
$\begin{array}{l}\text { Abstract IDDF2018-ABS-0253 } \\
\text { Table } 1\end{array}$ & Performances of three \\
classification task & ESO/ & $\begin{array}{c}\text { STO/Small } \\
\text { intes }\end{array}$ & $\begin{array}{c}\text { ESO/STO/Small } \\
\text { intes }\end{array}$ \\
\hline STO & $99.22 \%$ & $98.8 \%$ \\
Accuracy & $98.91 \%$ & $99.17 \%$ & - \\
Specific & $99.78 \%$ & $99.23 \%$ & -
\end{tabular}

$\mathrm{ESO}=$ Oesophagus $\mathrm{STO}=$ Stomach Small intes=Small Intestine

We do not classify oesophagus vs. small intestine because of the order of WCE passing through the human gut. Due to the intestinal of patients are not clean enough, some images were fulfilled with food residue or faecal residue, which leads to the inability to determine its location. Rest of the misclassified samples are almost located at the junction of two parts because the intestinal wall of these places contains the characteristics of the front and back parts.

Conclusions The CNNs show the great ability to distinguish the WCE images belongs to the oesophagus, stomach or small intestine. Some misclassified results were corrected based on the continuity of intestine for more robust performance, which will benefit the WCE abnormal automatic detection system behind. 